\title{
Vibration Control in MEMS Resonator Using Positive Position Feedback (PPF) Controller
}

\author{
Y.A. Amer, A.T. EL-Sayed, A.M. Salem \\ Department of Mathematics, Faculty of Science, Zagazig University, Zagazig, Egypt \\ yaser31270@yahoo.com \\ Department of Basic Sciences, Modern Academy for Engineering and Technology, Maadi, Egypt \\ ashraftaha211@yahoo.com \\ Department of Basic Sciences, Modern Academy for Engineering and Technology, Maadi, Egypt \\ amirasalim_math@yahoo.com
}

\begin{abstract}
In this paper, the vibration of a micro-electromechanical resonator with positive position feedback controller is studied. The analytical results are obtained to the first order approximation by using the multiple scale perturbation technique. The stability of the steady-state solution is presented and studied applying frequency response equations near the simultaneous primary and internal resonance cases. The effects of the controller and some system parameters on the vibrating system are studied numerically. The main result of this paper indicates that it is possible to reduce the vibration for the resonator system.
\end{abstract}

\section{Keywords}

MEMS Resonators; multiple scales perturbation; the simultaneous primary resonance case; PPF controller; stability

\section{INTRODUCTION}

In the area of mechanics and electronics, vibration controls of nonlinear parametrically excited system have been studied and illustrated in many researchers. Microelectromechanical (MEM) is one of the important topics in scientific researches of this problem. Siewe and Hegazy [1] studied the homoclinic bifurcation and the chaotic dynamics of a micromechanical resonator with electrostatic forces. They used different active vibration controllers to reduce the vibration of the micromechanical resonator system. Shaw et al. [2] investigated the dynamics of MEMS oscillators as frequency filters by using parametric resonance. El-Ganaini et al. [3] reported that the positive position feedback (PPF) controller is one of the most effective tools for the vibration control for nonlinear dynamical systems. Warminski et al. [4] presented active suppression of nonlinear composite beam vibrations by specific control algorithms. Hegazy [5] discussed the vibration control of an electromechanical seismograph system with time-varying stiffness by using cubic velocity feedback.

Ahamed et al. [6] introduced dynamic compensation for control of a rotary wing unmanned aerial vehicle (UAV) applying positive position feedback controller. The Positive Position Feedback (PPF) controller applied for a flexible manipulator is presented by Shan et al. [7]. They presented several vibration modes in the control strategy taking into account a linear mathematical model of the plant. The (PPF) control has been compared with the algorithm of velocity feedback. An experimental study shows that only PPF algorithm is able to work properly while slewing process is realized. Amer and EL-Sayed, Eissa et. al., Kamel et. al. and EL-Sayed et. al. [8-13] studied the passive controller (absorber) under multi external and parametric excitations which used to reduce the vibration of the different non-linear dynamical system. ELSayed and Bauomy [14] applied different type of controllers as passive and active control to reduce the torsional vibration of the nonlinear dynamical system using MSPT up to the second order approximation. This system is subjected to multi parametric excitation forces and modeled by the coupled nonlinear differential equations.

The aim of this paper is to suppress the vibration in the micro-electromechanical resonator using the positive position feedback controller at simultaneous primary and internal resonance case.

The present paper is prepared as follows. The equations of the problem which describes the vibration of MEMS resonator with PPF controller are shown in Section 2. In Sections 3 and 4, the mathematical treatments using multiple scales perturbation analysis and the stability of the equilibrium solutions are given to present the analytical solution of a secondorder nonlinear ordinary differential equations. The numerical simulations for the system without control and with the positive position feedback controller using time history and frequency-response functions are presented in Section 5. Comparison between analytical and numerical results is illustrated in Sections 6. Concluding remarks are presented in Section 7.

\section{Equations of the problem}

The nonlinear ordinary differential equation which defines the non-dimensional equation of motion for the MEMS resonator is derived in Ref. [1] as 
$\ddot{x}+2 \varepsilon \mu \dot{x}+\omega_{1}^{2} x+\varepsilon \alpha_{3} x^{3}+\varepsilon \alpha_{5} x^{5}=\frac{\varepsilon F}{(1-x)^{2}} \sin (\Omega t)+\varepsilon \gamma\left(\frac{1}{(1-x)^{2}}-\frac{1}{(1+x)^{2}}\right)+\varepsilon \lambda_{1} F_{c}(t)$

Taking into account that $(1-x)^{-2}=1+2 x+3 x^{2}$ and $(1+x)^{-2}=1-2 x+3 x^{2}$

We present a second order nonlinear controller (PPF), which designed to control the vibration of the main system (MEMS resonator). Then, the equation commanding the dynamics of the controller (PPF) is indicated as

$\ddot{u}+2 \varepsilon \mu_{1} \omega_{2} \dot{u}+\omega_{2}^{2} u=\varepsilon \lambda_{2} F_{f}(t)$

We determine the control signal $F_{c}=u$ and the feedback signal $F_{f}=x$ so the closed loop system equations are

$$
\begin{aligned}
& \ddot{x}+2 \varepsilon \mu \dot{x}+\omega_{1}^{2} x+\varepsilon \alpha_{3} x^{3}+\varepsilon \alpha_{5} x^{5}=\frac{\varepsilon F}{(1-x)^{2}} \sin (\Omega t)+\varepsilon \gamma\left(\frac{1}{(1-x)^{2}}-\frac{1}{(1+x)^{2}}\right)+\varepsilon \lambda_{1} u \\
& \ddot{u}+2 \varepsilon \mu_{1} \omega_{2} \dot{u}+\omega_{2}^{2} u=\varepsilon \lambda_{2} x
\end{aligned}
$$

Where $\mu$ and $\mu_{1}$ are damping coefficient of the MEMS resonator and the PPF controller, $F$ and $\Omega$ are the forcing amplitude and the frequency of the system, $\omega_{1}$ and $\omega_{2}$ are natural frequencies of the main system and PPF controller, $\alpha_{3}, \alpha_{5}$ and $\gamma$ are non-linear parameters, $\lambda_{1}$ and $\lambda_{2}$ are gains and $\varepsilon$ is a small perturbation parameter.

Fig. 1 show that the block diagram which describing equations (3) and (4)

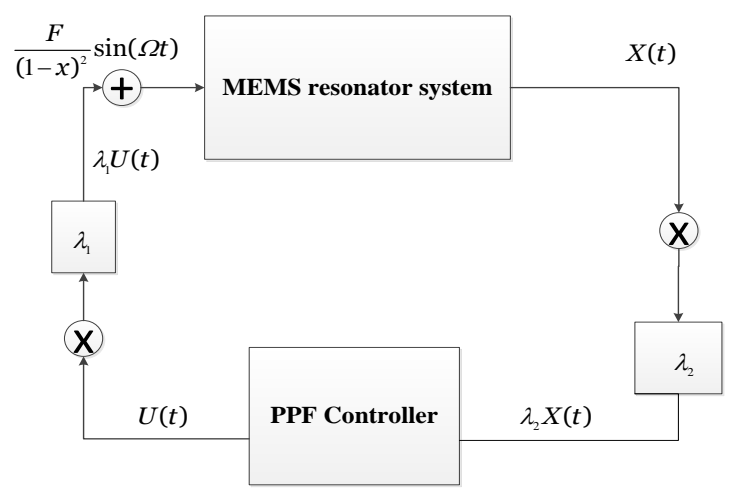

Fig. 1 Bock diagram of the closed loop system.

\section{Mathematical Treatments (MSPT)}

The multiple scales method is used to obtain the asymptotic first-order approximate solutions for (3) and (4) which assumed in the forms:

$$
x\left(T_{0}, T_{1}, \varepsilon\right)=x_{0}\left(T_{0}, T_{1}\right)+\varepsilon x_{1}\left(T_{0}, T_{1}\right)
$$


$u\left(T_{0}, T_{1}, \varepsilon\right)=u_{0}\left(T_{0}, T_{1}\right)+\varepsilon u_{1}\left(T_{0}, T_{1}\right)$

Where $\varepsilon$ is a small perturbation parameter $(0<\varepsilon<1), T_{0}=t$ and $T_{1}=\varepsilon t$ are the fast and slow time scales, respectively. The time derivatives became

$\frac{d}{d t}=D_{0}+\varepsilon D_{1}+\cdots$

$\frac{d^{2}}{d t^{2}}=D_{0}^{2}+2 \varepsilon D_{0} D_{1}+\ldots$

Where $D_{j}=\frac{\partial}{\partial T_{j}}, j=0,1$

Substituting (5) to (8) into (3) and (4), and equating the coefficients of equal power of $\varepsilon$ lead to:

$O\left(\varepsilon^{0}\right):$

$\left(D_{0}^{2}+\omega_{1}^{2}\right) x_{0}=0$

$\left(D_{0}^{2}+\omega_{2}^{2}\right) u_{0}=0$

$O(\varepsilon):$

$\left(D_{0}^{2}+\omega_{1}^{2}\right) x_{1}=-2 D_{0} D_{1} x_{0}-2 \mu D_{0} x_{0}-\alpha_{3} x_{0}^{3}-\alpha_{5} x_{0}^{5}+F\left(1+2 x_{0}+3 x_{0}^{2}\right) \sin (\Omega t)+\gamma\left(4 x_{0}\right)$

$$
+\lambda_{1} u_{0}
$$

$\left(D_{0}^{2}+\omega_{2}^{2}\right) u_{1}=-2 D_{0} D_{1} u_{0}-2 \mu_{1} \omega_{2} D_{0} u_{0}+\lambda_{2} x_{0}$

The general solution of equations (9) and (10) can be written in the following forms:

$x_{0}\left(T_{0}, T_{1}\right)=A_{1}\left(T_{1}\right) \exp \left(i \omega_{1} T_{0}\right)+c c$.

$u_{0}\left(T_{0}, T_{1}\right)=A_{2}\left(T_{1}\right) \exp \left(i \omega_{2} T_{0}\right)+c c$.

Where $A_{m}\left(T_{1}\right)(m=1,2)$ are unknown complex function in $T_{1}$ and $c c$ denotes the complex conjugate of the previous terms.

Substituting equations (13) and (14) into equations (11) and (12), we get the following:

$$
\begin{aligned}
\left(D_{0}^{2}+\omega_{1}^{2}\right) x_{1} & =\left[-2 i \omega_{1} D_{1} A_{1}-2 \mu i \omega_{1} A_{1}-3 \alpha_{3} A_{1}^{2} \bar{A}_{1}-10 \alpha_{5} A_{1}^{3} \bar{A}_{1}^{2}+4 \gamma A_{1}\right] \exp \left(i \omega_{1} T_{0}\right) \\
& +\left[-\alpha_{3} A_{1}^{3}-5 \alpha_{5} A_{1}^{4} \bar{A}_{1}\right] \exp \left(3 i \omega_{1} T_{0}\right)+\left[-\alpha_{5} A_{1}^{5}\right] \exp \left(5 i \omega_{1} T_{0}\right)+\left[\lambda_{1} A_{2}\right] \exp \left(i \omega_{2} T_{0}\right) \\
- & {\left[\frac{i F}{2}\left(1+6 A_{1} \bar{A}_{1}\right)\right] \exp \left(i \Omega T_{0}\right)-i F A_{1} \exp \left(i\left(\Omega+\omega_{1}\right) T_{0}\right)-i F \bar{A}_{1} \exp \left(i\left(\Omega-\omega_{1}\right) T_{0}\right) } \\
- & \frac{3 i F A_{1}^{2}}{2} \exp \left(i\left(\Omega+2 \omega_{1}\right) T_{0}\right)-\frac{3 i F \bar{A}_{1}^{2}}{2} \exp \left(i\left(\Omega-2 \omega_{1}\right) T_{0}\right)+c c . \\
\left(D_{0}^{2}+\omega_{2}^{2}\right) u_{1}= & {\left[-2 i \omega_{2} D_{1} A_{2}-2 \mu_{1} i \omega_{2}^{2} A_{2}\right] \exp \left(i \omega_{2} T_{0}\right)+\lambda_{2} A_{1} \exp \left(i \omega_{1} T_{0}\right)+c c . }
\end{aligned}
$$

For bounded solutions of equations (15) and (16), the secular terms, should be eliminated then the particular solutions of these equations are obtained as follows: 


$$
\begin{aligned}
x_{1} & =E_{1} \exp \left(3 i \omega_{1} T_{0}\right)+E_{2} \exp \left(5 i \omega_{1} T_{0}\right)+E_{3} \exp \left(i \omega_{2} T_{0}\right)+E_{4} \exp \left(i \Omega T_{0}\right)+E_{5} \exp \left(i\left(\Omega+\omega_{1}\right) T_{0}\right) \\
& +E_{6} \exp \left(i\left(\Omega-\omega_{1}\right) T_{0}\right)+E_{7} \exp \left(i\left(\Omega+2 \omega_{1}\right) T_{0}\right)+E_{8} \exp \left(i\left(\Omega-2 \omega_{1}\right) T_{0}\right)+c c . \\
u_{1} & =E_{9} \exp \left(i \omega_{1} T_{0}\right)+c c .
\end{aligned}
$$

Where $E_{n} \quad(n=1,2, \ldots, 9)$ are unknown complex function in $T_{1}$. (See Appendix)

\section{Stability Analysis}

In this paper, the case of the simultaneous primary and internal resonance case $\left(\Omega=\omega_{1}, \omega_{1}=\omega_{2}\right)$, which is the worst resonance case, is considered to study the stability of the system of equations (3) and (4). Introducing the detuning parameters $\sigma_{1}$ and $\sigma_{2}$ according to:

$$
\Omega=\omega_{1}+\varepsilon \sigma_{1} \text { and } \omega_{2}=\omega_{1}+\varepsilon \sigma_{2}
$$

Substituting equation (19) into equations (15) and (16) and eliminating the secular terms, leads to the solvability conditions for the first order approximation, hence the following differential equations are obtained:

$$
\begin{gathered}
2 i \omega_{1} D_{1} A_{1}=\left[-2 \mu i \omega_{1} A_{1}-3 \alpha_{3} A_{1}^{2} \bar{A}_{1}-10 \alpha_{5} A_{1}^{3} \bar{A}_{1}^{2}+4 \gamma A_{1}\right]+\left[\lambda_{1} A_{2}\right] \exp \left(i \sigma_{2} T_{1}\right) \\
-\left[\frac{i F}{2}\left(1+6 A_{1} \bar{A}_{1}\right)\right] \exp \left(i \sigma_{1} T_{1}\right)+\left[\frac{3 i F A_{1}^{2}}{2}\right] \exp \left(-i \sigma_{1} T_{1}\right)
\end{gathered}
$$

$2 i \omega_{2} D_{1} A_{2}=\left[-2 \mu_{1} i \omega_{2}^{2} A_{2}\right]+\left[\lambda_{2} A_{1}\right] \exp \left(-i \sigma_{2} T_{1}\right)$

To analyze the solution of equations (20) and (21), it is convenient to express $A_{m}\left(T_{1}\right)$ in the polar form as:

$A_{m}=\frac{1}{2} a_{m} \exp \left(i \varphi_{m}\right)$

where $a_{m}$ and $\varphi_{m}$ are real and represent both the steady state amplitudes and phases of motions, respectively. Inserting equation (22) into equations (19) and (21) then equating the real and the imaginary parts, we get the following:

$$
\begin{aligned}
& \dot{a}_{1}=-\mu a_{1}+\frac{\lambda_{1}}{2 \omega_{1}} a_{2} \sin \left(\theta_{2}\right)+\left[-\frac{F}{2 \omega_{1}}-\frac{3 F}{8 \omega_{1}} a_{1}^{2}\right] \cos \left(\theta_{1}\right) \\
& \dot{\varphi}_{1} a_{1}=\frac{3}{8 \omega_{1}} \alpha_{3} a_{1}^{3}+\frac{10}{32 \omega_{1}} \alpha_{5} a_{1}^{5}-\frac{2}{\omega_{1}} \gamma a_{1}-\frac{\lambda_{1}}{2 \omega_{1}} a_{2} \cos \left(\theta_{2}\right)+\left[-\frac{F}{2 \omega_{1}}-\frac{9 F}{8 \omega_{1}} a_{1}^{2}\right] \sin \left(\theta_{1}\right)
\end{aligned}
$$

$\dot{a}_{2}=-\mu_{1} \omega_{2} a_{2}-\frac{\lambda_{2}}{2 \omega_{2}} a_{1} \sin \left(\theta_{2}\right)$

$\dot{\varphi}_{2} a_{2}=-\frac{\lambda_{2}}{2 \omega_{2}} a_{1} \cos \left(\theta_{2}\right)$

where $\theta_{1}=\sigma_{1} T_{1}-\varphi_{1}, \theta_{2}=\sigma_{2} T_{1}+\varphi_{2}-\varphi_{1}$

By differentiating equation (27) with respect to $t$ we can eliminate $\dot{\varphi}_{1}$ and $\dot{\varphi}_{2}$ from equations (24) and (26) as the following:

$$
\dot{\varphi}_{1}=\sigma_{1}-\dot{\theta}_{1}, \dot{\varphi}_{2}=\left(\sigma_{1}-\sigma_{2}\right)+\left(\dot{\theta}_{2}-\dot{\theta}_{1}\right)
$$


From equations (23) to (26) and (28), the amplitude and phase modulating equations are

$$
\begin{aligned}
& \dot{a}_{1}=-\mu a_{1}+\frac{\lambda_{1}}{2 \omega_{1}} a_{2} \sin \left(\theta_{2}\right)+\left[-\frac{F}{2 \omega_{1}}-\frac{3 F}{8 \omega_{1}} a_{1}^{2}\right] \cos \left(\theta_{1}\right) \\
& \dot{\theta}_{1}=\sigma_{1}-\frac{3}{8 \omega_{1}} \alpha_{3} a_{1}^{2}-\frac{10}{32 \omega_{1}} \alpha_{5} a_{1}^{4}+\frac{2}{\omega_{1}} \gamma+\frac{\lambda_{1}}{2 \omega_{1} a_{1}} a_{2} \cos \left(\theta_{2}\right)-\left[-\frac{F}{2 \omega_{1} a_{1}}-\frac{9 F}{8 \omega_{1}} a_{1}\right] \sin \left(\theta_{1}\right) \\
& \dot{a}_{2}=-\mu_{1} \omega_{2} a_{2}-\frac{\lambda_{2}}{2 \omega_{2}} a_{1} \sin \left(\theta_{2}\right) \\
& \dot{\theta}_{2}=\sigma_{2}-\frac{3}{8 \omega_{1}} \alpha_{3} a_{1}^{2}-\frac{10}{32 \omega_{1}} \alpha_{5} a_{1}^{4}+\frac{2}{\omega_{1}} \gamma+\left[-\frac{\lambda_{2} a_{1}}{2 \omega_{2} a_{2}} \cos \left(\theta_{2}\right)+\frac{\lambda_{1} a_{2}}{2 \omega_{1} a_{1}}\right] \cos \left(\theta_{2}\right)-\left[-\frac{F}{2 \omega_{1} a_{1}}-\frac{9 F}{8 \omega_{1}} a_{1}\right] \sin \left(\theta_{1}\right)
\end{aligned}
$$

The steady-state solution of our dynamical system corresponding to the fixed point of equations (29) to (32) is obtained when

$\dot{a}_{m}=0, \dot{\theta}_{m}=0$

Inserting equation (33) into equations (29) to (32) we obtain:

$$
\begin{aligned}
& \mu a_{1}=\frac{\lambda_{1}}{2 \omega_{1}} a_{2} \sin \left(\theta_{2}\right)+\left[-\frac{F}{2 \omega_{1}}-\frac{3 F}{8 \omega_{1}} a_{1}^{2}\right] \cos \left(\theta_{1}\right) \\
& \sigma_{1} a_{1}-\left[\frac{3}{8 \omega_{1}} \alpha_{3} a_{1}^{3}+\frac{10}{32 \omega_{1}} \alpha_{5} a_{1}^{5}-\frac{2}{\omega_{1}} \gamma a_{1}\right]=-\frac{\lambda_{1}}{2 \omega_{1}} a_{2} \cos \left(\theta_{2}\right)+\left[-\frac{F}{2 \omega_{1}}-\frac{9 F}{8 \omega_{1}} a_{1}^{2}\right] \sin \left(\theta_{1}\right) \\
& \mu_{1} \omega_{2} a_{2}=-\frac{\lambda_{2}}{2 \omega_{2}} a_{1} \sin \left(\theta_{2}\right) \\
& \left(\sigma_{1}-\sigma_{2}\right) a_{2}=-\frac{\lambda_{2}}{2 \omega_{2}} a_{1} \cos \left(\theta_{2}\right)
\end{aligned}
$$

Squaring and adding equations (36) and (37) we get the first frequency response equation:

$\mu_{1}^{2} \omega_{2}^{2} a_{2}^{2}+\left(\sigma_{1}-\sigma_{2}\right)^{2} a_{2}^{2}=\frac{\lambda_{2}^{2}}{4 \omega_{2}^{2}} a_{1}^{2}$

From equations (36) and (37) we have:

$$
\begin{aligned}
& \sin \left(\theta_{2}\right)=\frac{-2 \mu_{1} \omega_{2}^{2} a_{2}}{\lambda_{2} a_{1}} \\
& \cos \left(\theta_{2}\right)=\frac{-2\left(\sigma_{1}-\sigma_{2}\right) \omega_{2} a_{2}}{\lambda_{2} a_{1}}
\end{aligned}
$$

Inserting equations (39) and (40) into equations (34) and (35)

$$
\mu a_{1}+\frac{\mu_{1} \omega_{2}^{2} a_{2}^{2} \lambda_{1}}{\lambda_{2} a_{1} \omega_{1}}=\left[-\frac{F}{2 \omega_{1}}-\frac{3 F}{8 \omega_{1}} a_{1}^{2}\right] \cos \left(\theta_{1}\right)
$$


$\sigma_{1} a_{1}-\left[\frac{3}{8 \omega_{1}} \alpha_{3} a_{1}^{3}+\frac{10}{32 \omega_{1}} \alpha_{5} a_{1}^{5}-\frac{2}{\omega_{1}} \gamma a_{1}\right]-\frac{\left(\sigma_{1}-\sigma_{2}\right) \omega_{2} a_{2}^{2} \lambda_{1}}{\lambda_{2} a_{1} \omega_{1}}=\left[-\frac{F}{2 \omega_{1}}-\frac{9 F}{8 \omega_{1}} a_{1}^{2}\right] \sin \left(\theta_{1}\right)$

Squaring and adding equations (41) and (42) we get the second frequency response equation:

$$
\begin{aligned}
& {\left[\mu a_{1}+\frac{\left.\mu_{1} \omega_{2}^{2} a_{2}^{2} \lambda_{1}\right]_{2} a_{1} \omega_{1}}{2}\left[-\frac{F}{2 \omega_{1}}-\frac{9 F}{8 \omega_{1}} a_{1}^{2}\right]^{2}+\left[\sigma_{1} a_{1}-\left[\frac{3}{8 \omega_{1}} \alpha_{3} a_{1}^{3}+\frac{10}{32 \omega_{1}} \alpha_{5} a_{1}^{5}-\frac{2}{\omega_{1}} \gamma a_{1}\right]-\frac{\left(\sigma_{1}-\sigma_{2}\right) \omega_{2} a_{2}^{2} \lambda_{1}}{\lambda_{2} a_{1} \omega_{1}}\right]^{2}\left[-\frac{F}{2 \omega_{1}}-\frac{3 F}{8 \omega_{1}} a_{1}^{2}\right]^{2}\right.} \\
& =\left[-\frac{F}{2 \omega_{1}}-\frac{9 F}{8 \omega_{1}} a_{1}^{2}\right]^{2}\left[-\frac{F}{2 \omega_{1}}-\frac{3 F}{8 \omega_{1}} a_{1}^{2}\right]^{2}
\end{aligned}
$$

To determine the stability of the nonlinear solution, one lets

$$
a_{m}=a_{m 0}+a_{m 1}, \quad \theta_{m}=\theta_{m 0}+\theta_{m 1}
$$

where $a_{m 0}$ and $\theta_{m 0}$ are the solutions of equations (29)-(32) and $a_{m 1}, \theta_{m 1}$ are perturbations which are assumed to be small compared to $a_{m 0}$ and $\theta_{m 0}$. Substituting equation (44) into equations (29)-(32) and keeping only the linear terms in $a_{m 1}$ and $\theta_{m 1}$, we obtain that

$\dot{a}_{11}=\left(-\mu-\frac{3 F}{4 \omega_{1}} a_{10} \cos \theta_{10}\right) a_{11}+\left(\frac{F}{2 \omega_{1}} \sin \theta_{10}+\frac{3 F}{8 \omega_{1}} a_{10}^{2} \sin \theta_{10}\right) \theta_{11}+\left(\frac{\lambda_{1}}{2 \omega_{1}} \sin \theta_{20}\right) a_{21}+\left(\frac{\lambda_{1}}{2 \omega_{1}} a_{20} \cos \theta_{20}\right) \theta_{21}$

$\dot{\theta}_{11}=\left(\frac{\sigma_{1}}{a_{10}}-\frac{9}{8 \omega_{1}} \alpha_{3} a_{10}-\frac{50}{32 \omega_{1}} \alpha_{5} a_{10}^{3}+\frac{2}{\omega_{1} a_{10}} \gamma+\frac{9 F}{4 \omega_{1}} \sin \theta_{10}\right) a_{11}+\left(\frac{F}{2 a_{10} \omega_{1}} \cos \theta_{10}+\frac{9 F}{8 \omega_{1}} a_{10} \cos \theta_{10}\right) \theta_{11}$

$+\left(\frac{\lambda_{1}}{2 a_{10} \omega_{1}} \cos \theta_{20}\right) a_{21}+\left(\frac{-\lambda_{1}}{2 a_{10} \omega_{1}} a_{20} \sin \theta_{20}\right) \theta_{21}$

$\dot{a}_{21}=\left(-\frac{\lambda_{2}}{2 \omega_{2}} \sin \theta_{20}\right) a_{11}+\left(-\mu_{1} \omega_{2}\right) a_{21}+\left(-\frac{\lambda_{2}}{2 \omega_{2}} a_{10} \cos \theta_{20}\right) \theta_{21}$

$\dot{\theta}_{21}=\left(-\frac{\lambda_{2}}{2 a_{20} \omega_{2}} \cos \theta_{20}+\frac{\sigma_{1}}{a_{10}}-\frac{9}{8 \omega_{1}} \alpha_{3} a_{10}-\frac{50}{32 \omega_{1}} \alpha_{5} a_{10}^{3}+\frac{2}{\omega_{1} a_{10}} \gamma+\frac{9 F}{4 \omega_{1}} \sin \theta_{10}\right) a_{11}+\left(\frac{F}{2 a_{10} \omega_{1}} \cos \theta_{10}+\frac{9 F}{8 \omega_{1}} a_{10} \cos \theta_{10}\right) \theta_{11}$

$+\left(\frac{\sigma_{2}-\sigma_{1}}{a_{20}}+\frac{\lambda_{1}}{2 a_{10} \omega_{1}} \cos \theta_{20}\right) a_{21}+\left(\frac{\lambda_{2}}{2 a_{20} \omega_{2}} a_{10} \sin \theta_{20}-\frac{\lambda_{1}}{2 a_{10} \omega_{1}} a_{20} \sin \theta_{20}\right) \theta_{21}$

Equations (45) to (48) can be represented in the following matrix form:

$\left[\begin{array}{llll}\dot{a}_{11} & \dot{\theta}_{11} & \dot{a}_{21} & \dot{\theta}_{21}\end{array}\right]^{T}=[J]\left[\begin{array}{llll}a_{11} & \theta_{11} & a_{21} & \theta_{21}\end{array}\right]^{T}$

The eigenvalue equation of the Jacobian matrix $[J]$ can be obtained as

$\lambda^{4}+R_{1} \lambda^{3}+R_{2} \lambda^{2}+R_{3} \lambda+R_{4}=0$

where $\left(R_{1}, R_{2}, \ldots, R_{4}\right)$ are functions of the parameters $\left(a_{1}, a_{2}, \omega_{1}, \omega_{2}, \sigma_{1}, \sigma_{2}, \mu, \mu_{1}, \alpha_{3}, \alpha_{5}, \gamma, \lambda_{1}, \lambda_{2}, F, \theta_{1}, \theta_{2}\right)$. If the real part of each eigenvalue of the Jacobian matrix $[J]$ of the right-hand side of (45)-(48) is negative; otherwise, it is 
unstable. According to the Routh-Hurwitz criterion, the necessary and sufficient conditions for all the roots of equation (50) to have negative real parts are

$$
R_{1}>0, R_{1} R_{2}-R_{3}>0, R_{3}\left(R_{1} R_{2}-R_{3}\right)-R_{1}^{2} R_{4}>0, R_{4}>0
$$

\section{Numerical discussions}

In this section, the differential equations of the MEMS resonator without and with two PPF controller are solved numerically (applying Runge-Kutta 4th order method using MATLAB 7.0 software) at the simultaneous primary and internal resonance case. Fig. 2 and Fig. 3 show the results at the selected values $\left(\omega_{1}=1, \omega_{2}=\omega_{1}, \Omega_{1} \cong \omega_{1}, \mu=0.00005, \mu_{1}=0.005, \alpha_{3}=3.675 \times 10^{-5}, \alpha_{5}=1.3506 \times 10^{-9}, \gamma=0.044, \lambda_{1}=0.8\right.$, $\lambda_{2}=0.8, F=0.0087$ )

Fig. 2 shows that the response for MEMS resonator without PPF controller where the steady state amplitude ( $x$ ) is increased to about $1150 \%$ of the excitation force amplitude $(F)$ with multi limit cycles.

Fig. 3 illustrates the behavior of the MEMS resonator with PPF controller, where the steady state amplitude ( $x$ ) is reduced to about $2.3 \%$ of the excitation force amplitude $(F)$. The steady state amplitude of the PPF controller $(u)$ are about $115 \%$ of the excitation force amplitude $(F)$. It is worth to notice that from the Figs. 2 and 3 that the steady-state amplitude of the MEMS resonator with PPF controller was reduced to about $99.8 \%$ from its value without PPF controller. This means that the effectiveness of the controller $E_{a}\left(E_{a}=\right.$ steady state amplitude of the MEMS resonator without controller / steady state amplitude of the MEMS resonator with controller) is about 500 for the main system $(x)$.
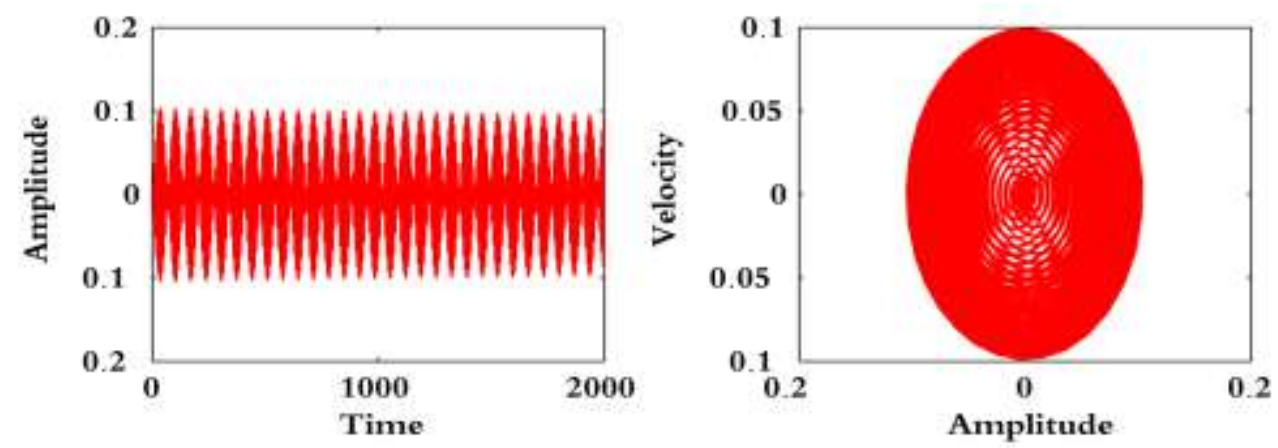

Fig. 2 Response of the MEMS resonator without controller at primary resonance case $\left(\Omega_{1} \cong \omega_{1}\right)$ 

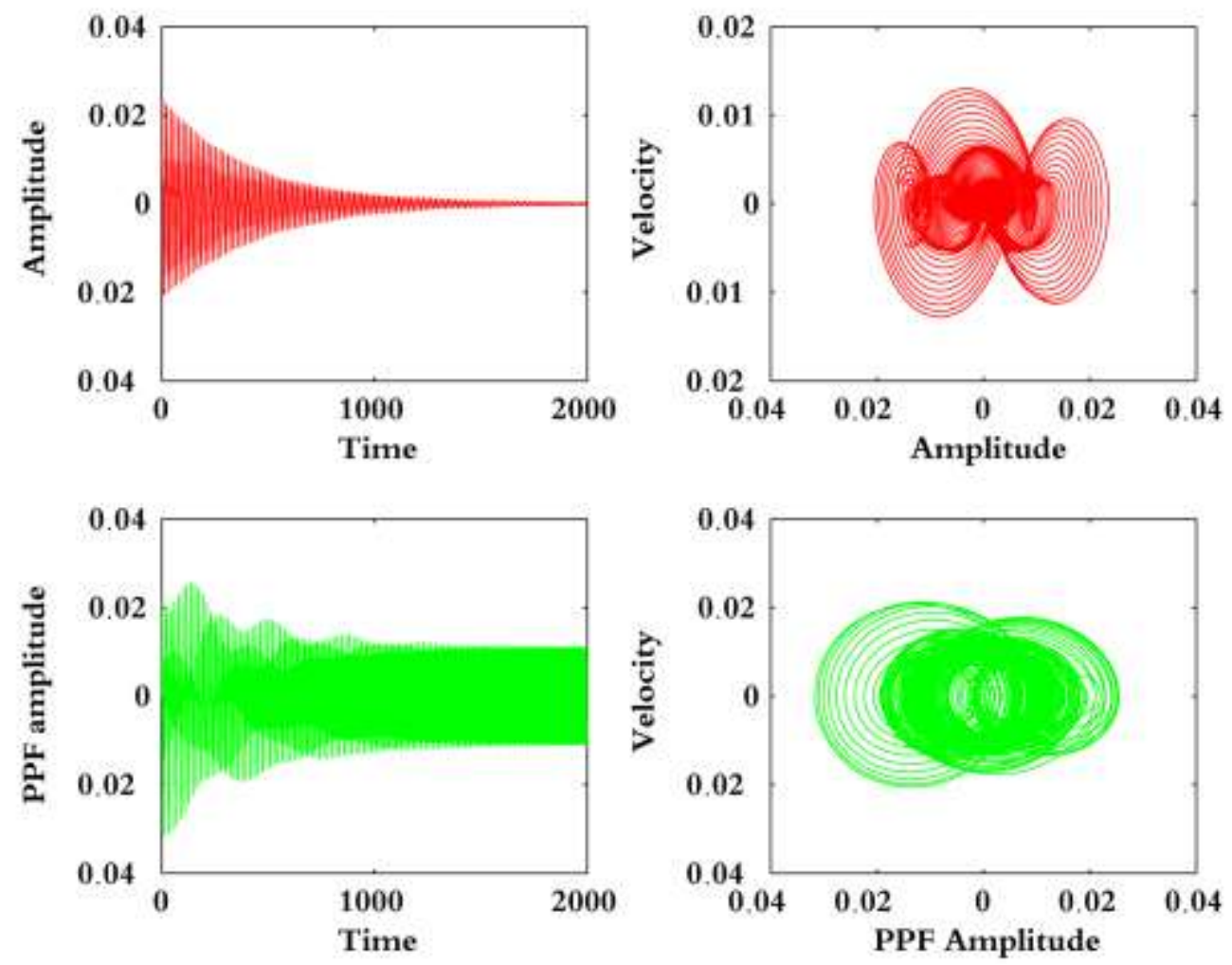

Fig. 3 Response of MEMS resonator with PPF controller at simultaneous primary an internal resonance case

$$
\left(\Omega_{1} \cong \omega_{1}, \omega_{2} \cong \omega_{1}\right)
$$

The effects of different parameters were investigated by solving the frequency response equations (38) and (43). The results are illustrated graphically in Figs. (4 to 14). From the obtained figures, the steady state amplitudes $a_{1}$ and $a_{2}$ are presented against detuning parameters $\sigma_{1}$ and $\sigma_{2}$ for the selected practical case $\left(a_{1} \neq 0, a_{2} \neq 0\right)$. The solid lines stand for the stable solution as shown in the figures.

Fig. 4 shows the frequency response curves of the main system with controller, where Fig. 4 a shows the frequency response curve for the MEMS resonator (main system) and Fig. $4 \mathrm{~b}$ shows the frequency-response curves for PPF controller. It is clear that the minimum steady-state amplitude of the main system $a_{1}$ occurs when $\sigma_{1}=0$.
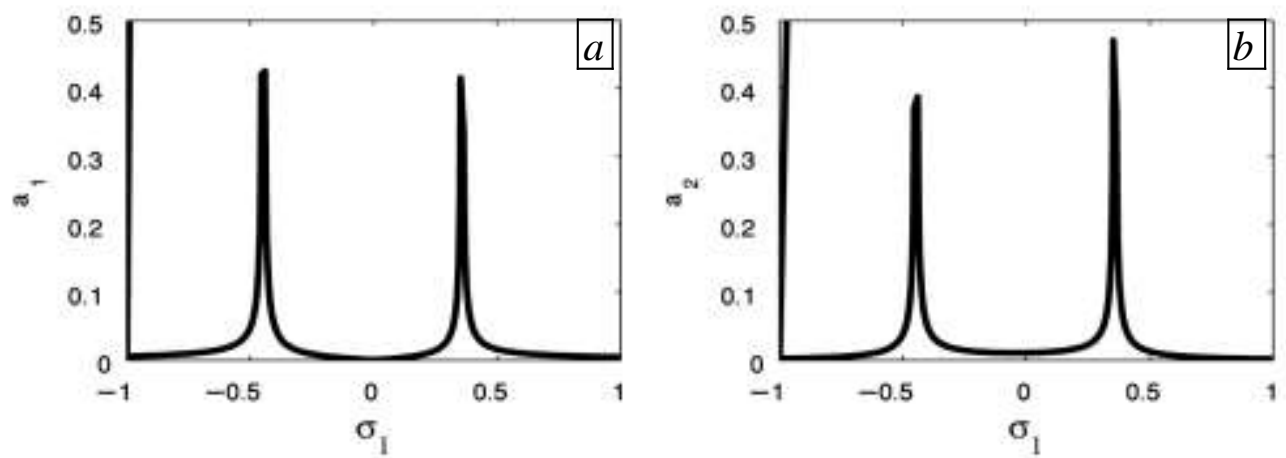

Fig. 4 Frequency-response curves of: (a) the main system $\left(a_{1}\right)$ and (b) the controller $\left(a_{2}\right)$

Fig. 5 shows that the steady state amplitudes for both the main system and the PPF controller are increased according to the increasing values of the excitation force amplitude $F$. 

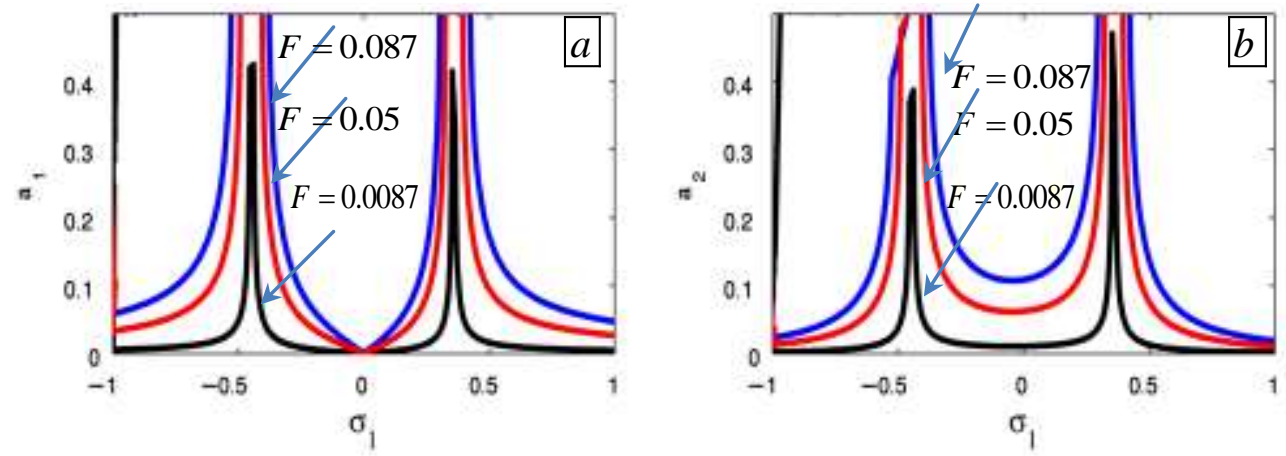

Fig. 5 Effect of the excitation force $F$ on: (a) the main system $\left(a_{1}\right)$ and (b) the controller $\left(a_{2}\right)$

Fig. 6 shows that when the PPF controller's damping coefficient $\mu_{1}$ values increases, the peak amplitudes of the main system and the PPF controller are decrease. Also, at Fig.7 we find the same effect of the system damping coefficient $\mu$.
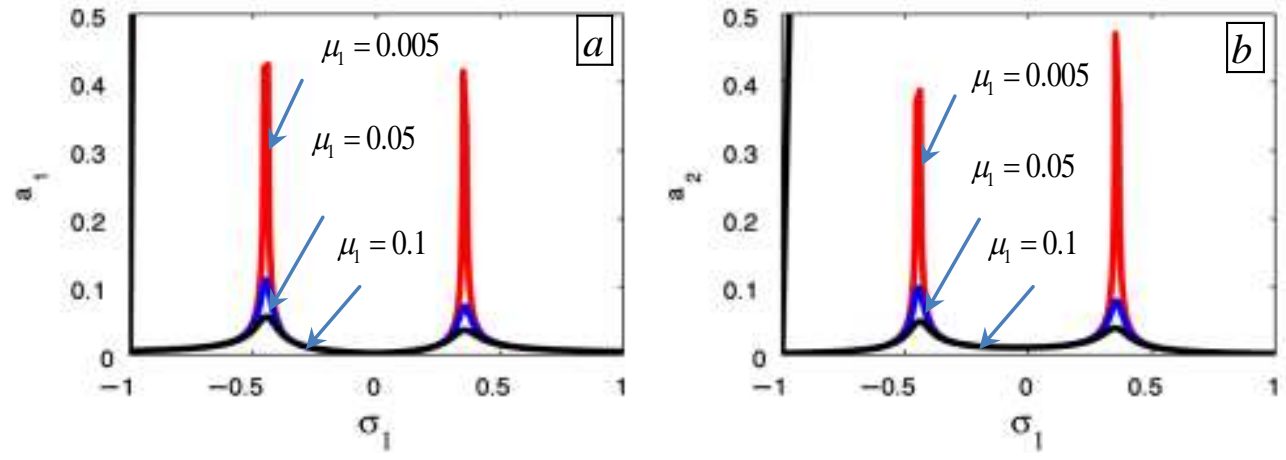

Fig. 6 Effect of the damping coefficients $\mu_{1}$ on: (a) the main system $\left(a_{1}\right)$, and (b) the controller $\left(a_{2}\right)$
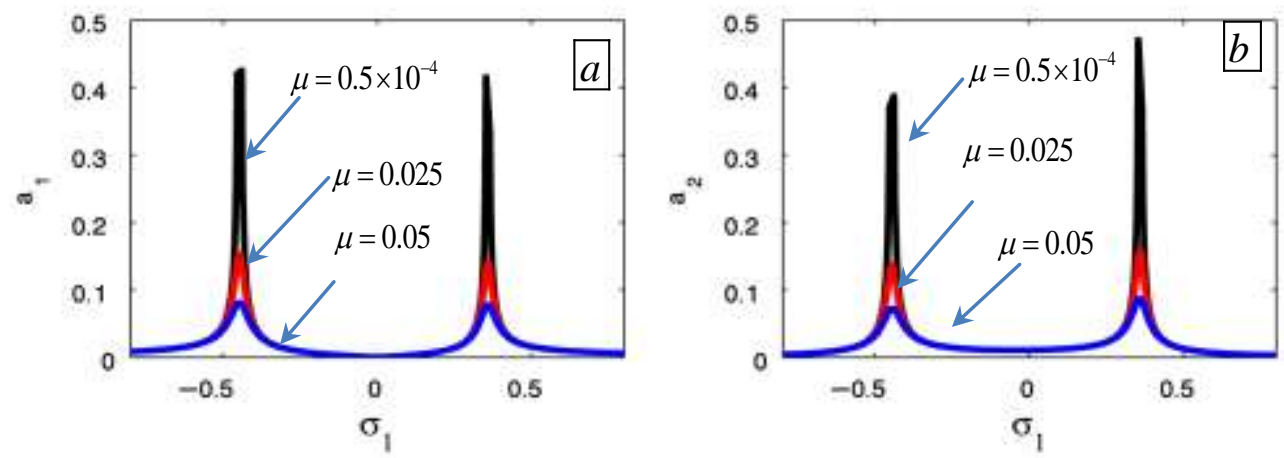

Fig. 7 Effect of the damping coefficients $\mu$ on: (a) the main system $\left(a_{1}\right)$, and (b) the controller $\left(a_{2}\right)$

Fig. 8 appears that for increasing values of the linear natural frequency of the main system $\omega_{1}$, the peak amplitudes of the main system and the PPF controller are decrease and the vibration reduction frequency bandwidth of the control for the amplitudes of the main system and the PPF controller are narrower. 

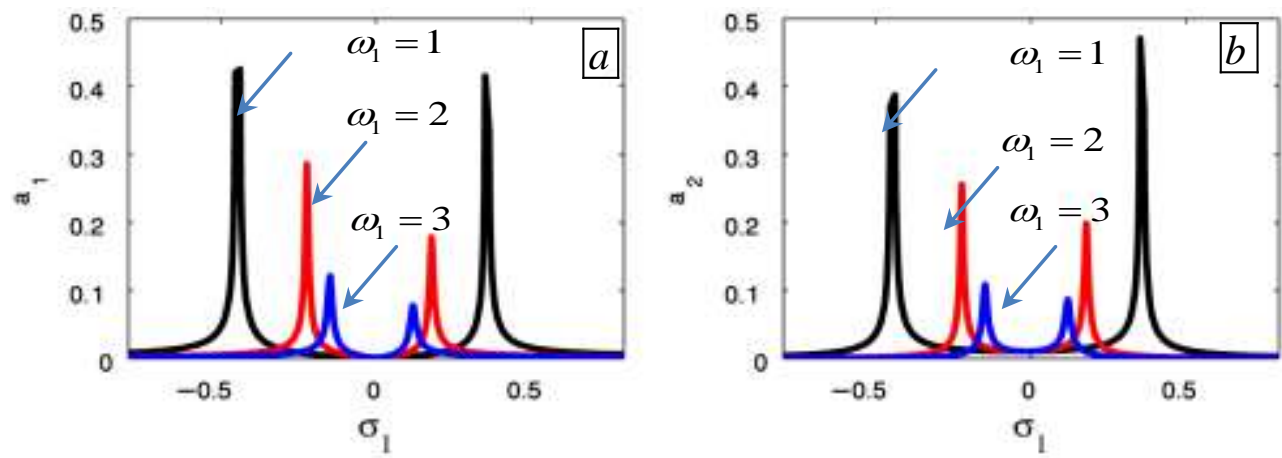

Fig. 8 Effect of the natural frequency $\omega_{1}$ on: (a) the main system $\left(a_{1}\right)$, and (b) the controller $\left(a_{2}\right)$

Fig. 9 illustrates that for decreasing and negative value of the nonlinear parameter $\gamma$, the curves are shifted to right and when it was positive with increasing value, the curves are shifted to left.
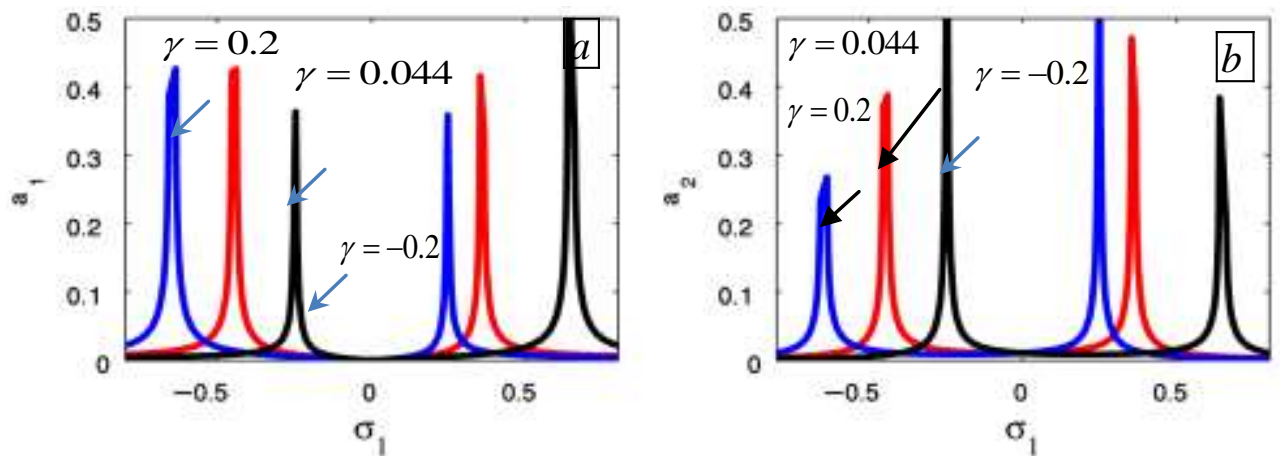

Fig. 9 Effect of the nonlinear parameter $\gamma$ on: (a) the main system $\left(a_{1}\right)$, and (b) the controller $\left(a_{2}\right)$

Figs. 10, 11 present the same effect of the feedback signal gain $\lambda_{1}, \lambda_{2}$. As shown in Fig. 10 and Fig. 11 for increasing values of $\lambda_{1}, \lambda_{2}$, the vibration reduction frequency bandwidth of the control for the amplitudes of the main system and the PPF controller are wider.
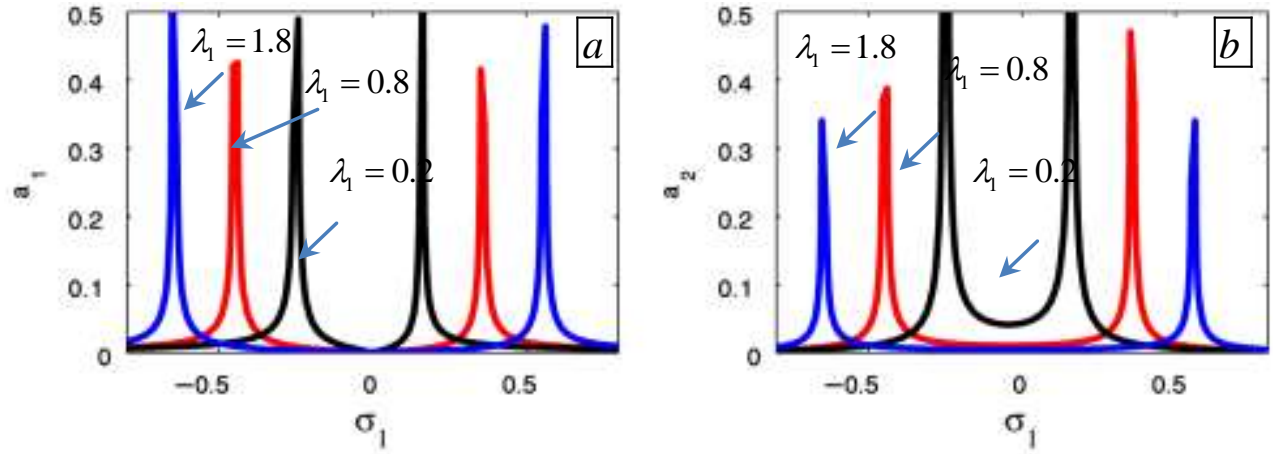

Fig. 10 Effect of the control signal gain $\lambda_{1}$ on: (a) the main system $\left(a_{1}\right)$, and (b) the controller $\left(a_{2}\right)$

Furthermore for increasing the value of nonlinear parameter $\alpha_{3}$, the amplitudes of the main system and PPF control are decreases with small shift to left as shown in Fig. 12. 

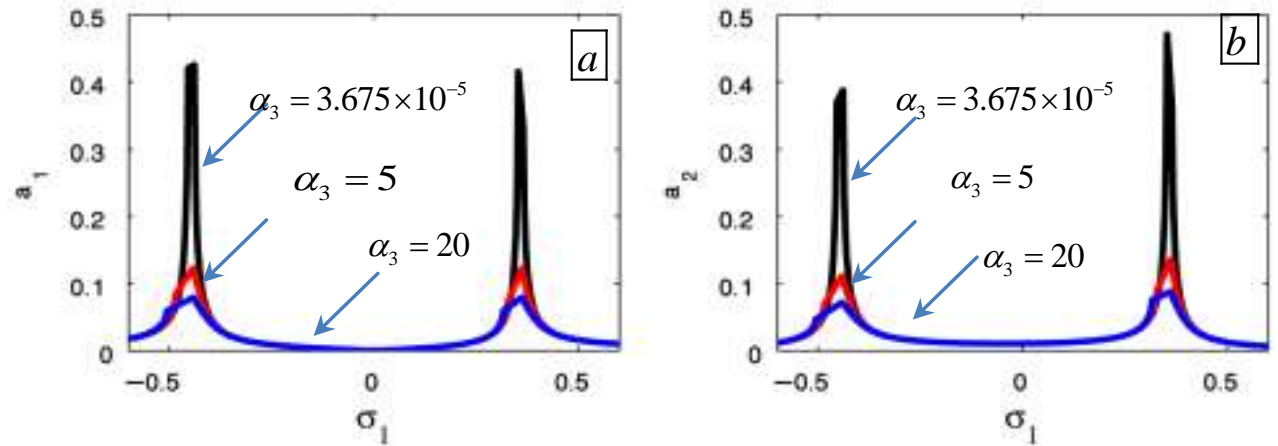

Fig. 12 Effect of the nonlinear parameter $\alpha_{3}$ on: (a) the main system $\left(a_{1}\right)$, and (b) the controller $\left(a_{2}\right)$

From Fig. 13(a) and (b), we found that the frequency response curve is bent to the right when $\alpha_{5}>1.3506 \times 10^{-9}$, indicating a hardening-type spring nonlinearity and bent to the left when $\alpha_{5}<1.3506 \times 10^{-9}$, indicating a softening-type spring nonlinearity.
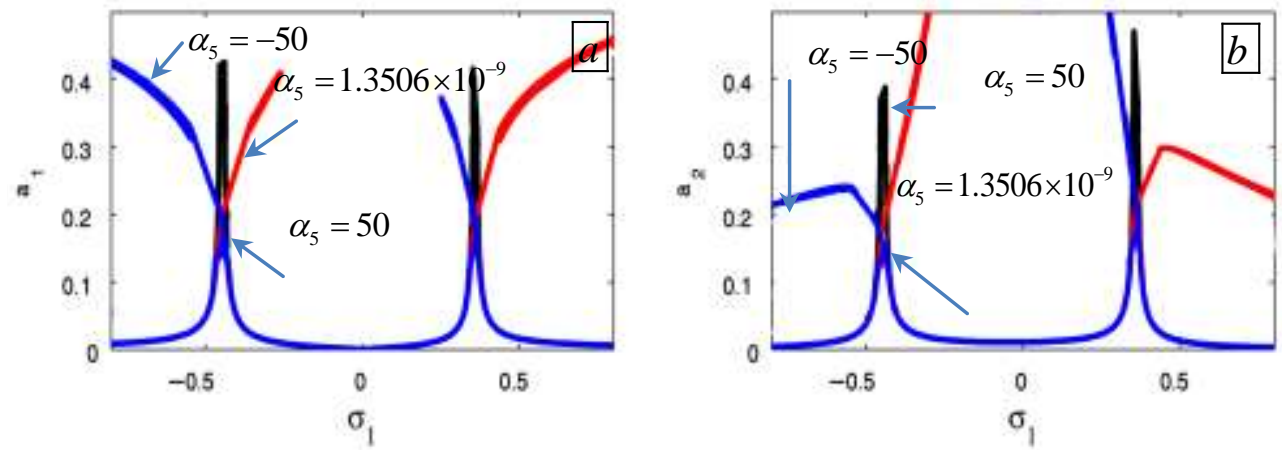

Fig. 13 Effect of the nonlinear parameter $\alpha_{5}$ on: (a) the main system $\left(a_{1}\right)$, and (b) the controller $\left(a_{2}\right)$

Fig. 14 shows the frequency response-curves of both the main system and the controller for three different values of the internal detuning parameter $\sigma_{2}$. Here, Fig. 14 show that for $\sigma_{2}=-0.5$, the minimum main system steady-state amplitude and the controller occur when $\sigma_{1}=-0.5$, for $\sigma_{2}=0$ the minimum main system steady-state amplitude and the controller occur when $\sigma_{1}=0$ and for $\sigma_{2}=0.5$, the minimum main system steady-state amplitude and the controller occur when $\sigma_{1}=0.5$. Based on Fig. 11, the minimum main system steady-state amplitude and the controller occur when $\sigma_{1}=\sigma_{2}$.
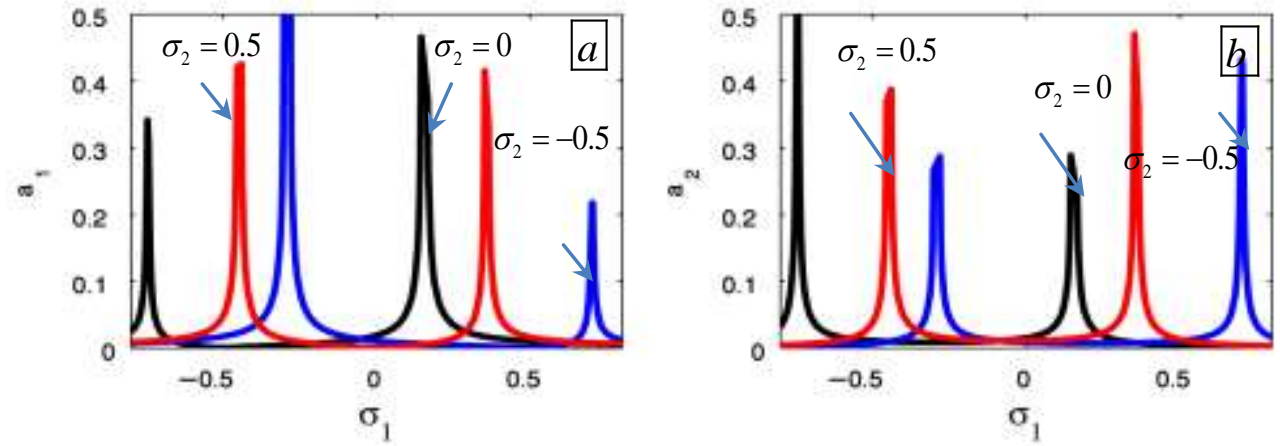

Fig. 14 Effect of the detuning parameter $\sigma_{2}$ on: (a) the main system $\left(a_{1}\right)$, and (b) the controller $\left(a_{2}\right)$ 


\section{Comparison between analytical and numerical solutions}

Fig. 15, represent the comparison with a good agreement between the numerical solution of equations (3-4) and analytical solution given by equations (29-32) for MEMS resonator with PPF controller for chosen values of the system parameters are presented graphically in Fig. 2. The dashed lines show the analytical solution and the continuous lines represent numerical solution.
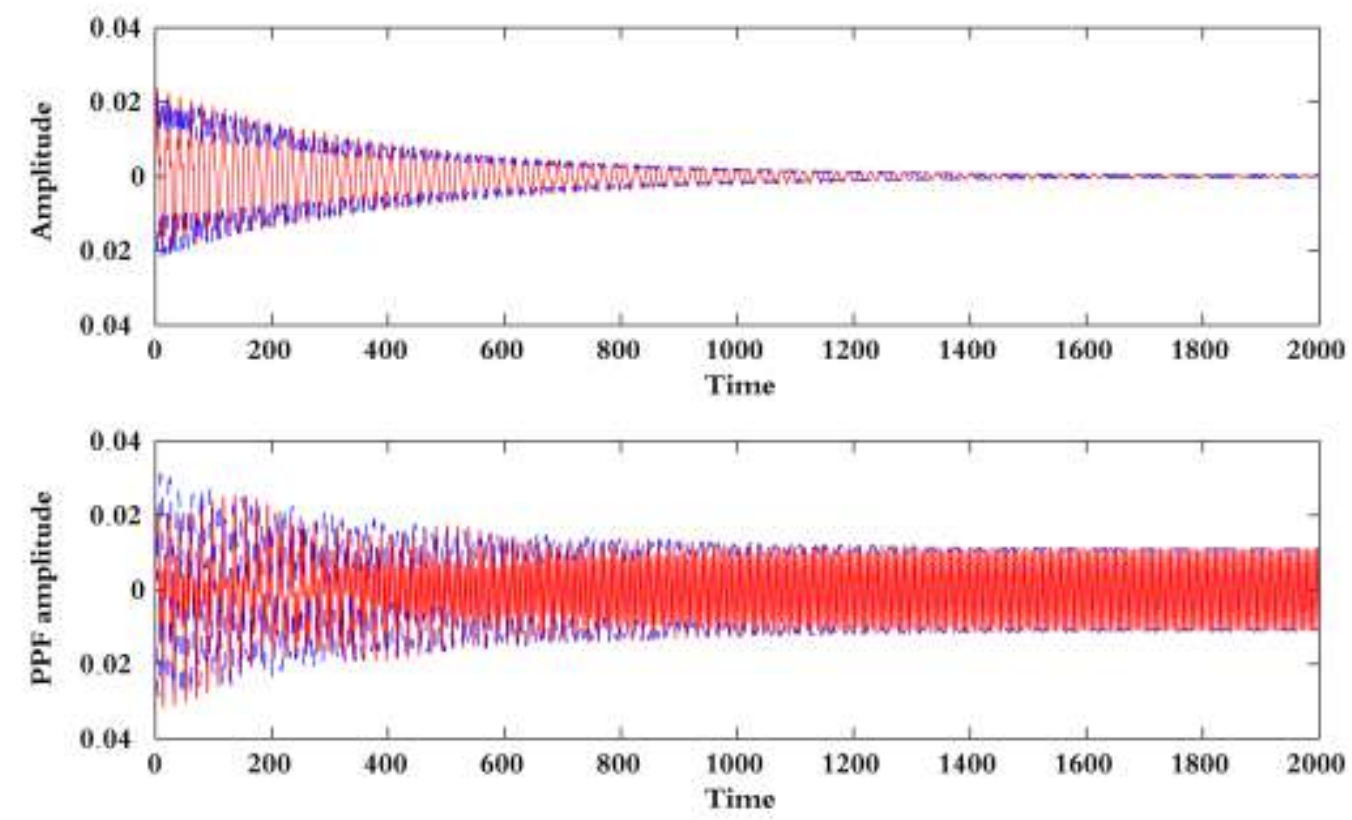

Fig. 15 Time response of: (a) MEMS resonator, and (b) PPF controller

\section{Conclusions}

The vibration in MEMS resonator is controlled with PPF controller. The system is studied near the simultaneous primary and internal resonance case by the method of multiple scales. The stability of the system under the simultaneous resonances is studied applying the frequency response equation method. The effects of the different parameters of the system and the controllers are studied numerically.

From the above study the results may be concluded:

1. The worst resonance cases of the system is the simultaneous resonance case: $\Omega \cong \omega_{1}$ and $\omega_{1} \cong \omega_{2}$.

2. The effectiveness of the controller $E_{a}$ are about 500 for $(x)$.

3. The vibration reduction controller frequency bandwidth may be wider as $\lambda_{1}, \lambda_{2}, \omega_{1}$ are increases.

4. The steady state amplitudes of the main system $(x)$ are monotonic increasing function to $F$.

5. The steady state amplitudes of the main system $(x)$ are monotonic decreasing function to $\mu, \mu_{1}$.

\section{References}

1. Siewe Siewe M. and Hegazy U. H.: Homoclinic bifurcation and chaos control in MEMS resonators, Applied Mathematical Modelling, 35, 5533-5552, 2011.

2. Shaw S., Turner K., Rhoads J. and Baskaranm R.: Parametrically excited MEMSIUTAM Symp. Caotic Dyn, Control Syst, Process 122, 37-146, 2003.

3. El-Ganaini W.A., Saeed N.A. and Eissa M.: Positive position feedback (PPF) controller for suppression of nonlinear system vibration, Nonlinear Dyn, 72, 517-537, 2013.

4. Warminski, J., Bochenski, M., Jarzyna, W., Filipek, P. and Augustyinak, M.: Active suppression of nonlinear composite beam vibrations by selected control algorithms. Commun. Nonlinear Sci. Numer. Simul. 16, 22372248, 2011.

5. Hegazy U.H.: Dynamics and control of a self-sustained electromechanical seismographs with time-varying stiffness, Meccanica, 44, 355-368, 2009.

6. Ahamed B. and Pota H.R.: Dynamic compensation for control of a rotary wing UAV using positive position feedback., J. Intell. Robot. Syst., 61, 43-56, 2011. 
7. Shan J., Liu H. and Sun D.: Slewing and vibration control of a single-link flexible manipulator by positive position feedback (PPF), Mechatronics, 15,487-503, 2005.

8. Amer Y. A. and EL-Sayed A. T.: Vibration suppression of nonlinear system via nonlinear absorber, Communication in Nonlinear Science and Numerical Simulation, 13, 1948-63, 2008.

9. Kamel M., Eissa M. and EL-Sayed A. T.: Vibration reduction of a non-linear spring pendulum under multiparametric excitations via a longitudinal absorber, Physica Scripta, 80, (12pp) 025005, 2009.

10. Eissa M., Kamel M. and EL-Sayed A. T.: Vibration reduction of multi parametric excited spring pendulum via a transversally tuned absorber, Nonlinear Dynamics, 61, 109-121, 2010.

11. EL-Sayed A. T., Kamel M. and Eissa M.: Vibration reduction of a pitch-roll ship model with longitudinal and transverse absorbers under multi excitations, Mathematical and Computer Modelling, 52, 1877-1898, 2010.

12. Eissa M., Kamel M. and EL-Sayed A. T.: Vibration reduction of a nonlinear spring pendulum under multi external and parametric excitations via a longitudinal absorber, Meccanica, 46, 325-340, 2011.

13. Eissa M., Kamel M. and EL-Sayed A. T.: Vibration suppression of a 4-DOF nonlinear spring pendulum via longitudinal and transverse absorbers, Journal of Applied Mechanics. ASME, 79(1), 011007 (11 pages), 2012.

14. EL-Sayed A. T and Bauomy H.S.: Vibration control of helicopter blade flapping via time-delay absorber, Meccanica, 49(3), 587-600, 2014.

\section{Appendix}

$$
\begin{aligned}
& E_{1}=\left[\frac{\alpha_{3} A_{1}^{3}+5 \alpha_{5} A_{1}^{4} \bar{A}_{1}}{8 \omega_{1}^{2}}\right] \\
& E_{2}=\left[\frac{\alpha_{5} A_{1}^{5}}{24 \omega_{1}^{2}}\right] \\
& E_{3}=\left[\frac{\lambda_{1} A_{2}}{\omega_{1}^{2}-\omega_{2}^{2}}\right] \\
& E_{3}=-\left[\frac{i F\left(1+6 A_{1} \bar{A}_{1}\right)}{2\left(\omega_{1}^{2}-\Omega^{2}\right)}\right] \\
& E_{5}=-\left[\frac{i F A_{1}}{\left(\omega_{1}^{2}-\left(\Omega+\omega_{1}\right)^{2}\right)}\right] \\
& E_{6}=-\left[\frac{i F \bar{A}_{1}}{\left(\omega_{1}^{2}-\left(\Omega-\omega_{1}\right)^{2}\right)}\right] \\
& E_{7}=-\left[\frac{3 i F A_{1}^{2}}{2\left(\omega_{1}^{2}-\left(\Omega+2 \omega_{1}\right)^{2}\right)}\right] \\
& E_{8}=-\left[\frac{3 i F \bar{A}_{1}^{2}}{2\left(\omega_{1}^{2}-\left(\Omega-2 \omega_{1}\right)^{2}\right)}\right] \\
& E_{9}=\left[\frac{\lambda_{2} A_{1}}{\omega_{2}^{2}-\omega_{1}^{2}}\right]
\end{aligned}
$$




\section{Author' biography with Photo}
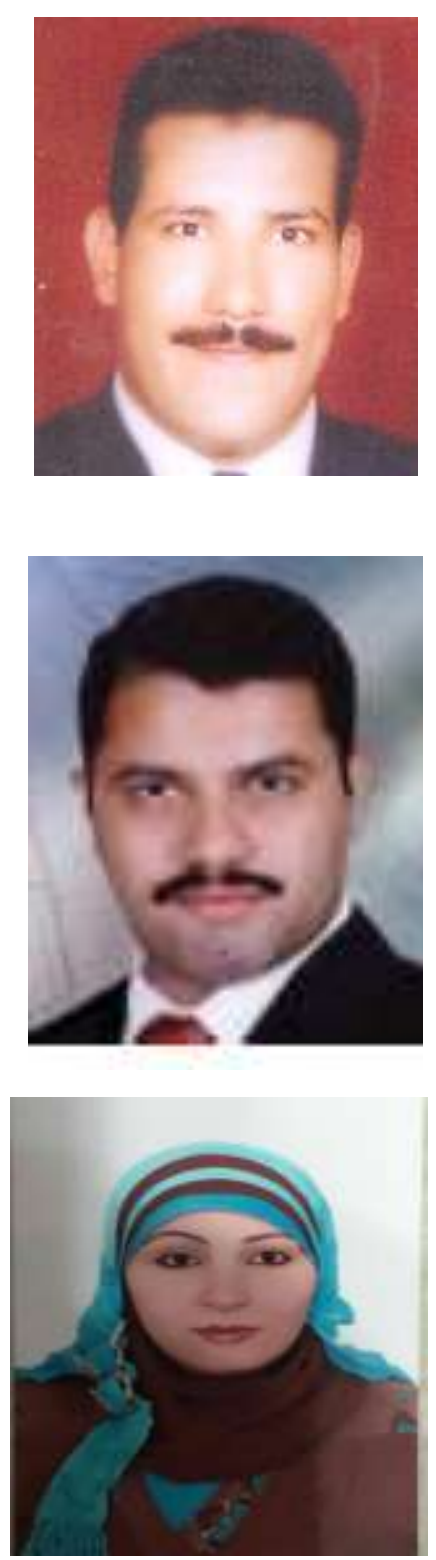

Y. A. Amer received his B.S. degree inMathematics from Zagazig University, EGYPT, in 1992. He then received his M.S.C and Ph.D. degrees from ZagazigUniversity, in 1996 and 2002, respectively. Dr. Y. A. Amer is currently a Professor of Mathematics at the Department of Mathematics, Faculty of Science, Zagaziga University, Egypt. Dr. Y. A. Amer research interests include Nonlinear dynamical systems, Numerical Analysis, Vibration control and Partial differential equations.

A. T. EL-Sayed received his B.S. degree in Mathematics from Zagazig University, EGYPT, in 2001. He then received his M.S.c and Ph.D. degrees from Zagazig University, in 2007 and 2011, respectively. Dr. A. T. EL-Sayed is currently an Assistant Professor of Mathematics at the Department of Basic Sciences, Modern academy for Engineering and Technology, Egypt. Dr. A. T. EL-Sayed research interests include Differential equations which simulates non-linear dynamical systems, Numerical Analysis and Vibration control.

A.M. Salem received her B.S. degree in Mathematics from AL-Azhar University, EGYPT, in 2004. She thenreceived her M.S.c.degrees from AL-Azhar University, in 2011. F. T. El-Bahrawy is currently an Assistant Lecture of Mathematics at the Department of Basic Sciences,Modern academy for Engineering and Technology, Egypt. A.M. Salem research interests include Differential equations, Numerical Analysis, and Vibration control. 